\title{
Update of the observation system for the primary energy spectrum with compact EAS arrays in LAAS experiment
}

\author{
Hiroki Matsumoto ${ }^{* a}$, Atsushi lyono $^{b}$, Kazuhide Okei $^{a}$, Shuhei Tsuji ${ }^{a}$, Soji Ohara ${ }^{c}$, \\ Nobuaki Ochi ${ }^{d}$, Nobusuke Takahashi ${ }^{e}$, Takao Nakatsuka ${ }^{g}$ and Saya Yamamoto ${ }^{h}$, \\ ${ }^{a}$ Kawasaki Medical School \\ Kurashiki 701-0192, Japan \\ ${ }^{b}$ Dept. of Fundamental Science, Faculty of Science, Okayama university of Science \\ Okayama 700-0005, Japan \\ ${ }^{c}$ Nara Sangyo University \\ Nara 636-8503, Japan \\ ${ }^{d}$ Toyo University \\ Tokyo 112-8606, Japan \\ ${ }^{e}$ Dept. of Physics, Hirosaki University \\ Hirosaki 036-8560, Japan \\ ${ }^{g}$ Okayama Shoka University \\ Okayama 700-8601, Japan \\ ${ }^{h}$ Dept. of Fundamental Science, Okayama University of Science \\ Okayama 700-0005, Japan \\ E-mail: h.mat sumoto@med.kawasaki-m.ac.jp iyono@das.ous.ac.jp
}

\begin{abstract}
The relation between the core distance and the time structure of extensive air shower (EAS) particles shown by Linsley provided to determine the primary energy by using compact EAS arrays. In an apparatus for restricting the zenith angle of EAS event, relocation and update of the apparatus was carried out. And the simulation for this upgrading was performed. We report the simulation results and comparisons of old and new apparatus.
\end{abstract}

35th International Cosmic Ray Conference - ICRC2017

10-20 July, 2017

Bexco, Busan, Korea

\footnotetext{
* Speaker.
} 


\section{Introduction}

Large Area Air Shower (LAAS) experiment 10 , 2 has been studied and observed in cosmic ray phenomena by using compact Extensive Air Shower (EAS) arrays installed in several sites since 1996. The distance between each site is about several $\mathrm{km} \sim 900 \mathrm{~km}$, and observation time is synchronized by a Global Positioning System (GPS). The mainly purpose of LAAS experiment is research in EAS events of long-baseline correlation. The cause of long-baseline correlation events is predicted to be Gerasimova-Zatsepin effect [3, ultra-high-energy $\gamma$-rays or exotic phenomena. And moreover, the anisotropy and the primary energy spectrum of high energy cosmic rays have been reported 4, 5, 6, , 7, 8. In the primary energy spectrum, the integral primary energy spectrum in its energy range of around $10^{14} \mathrm{eV}$ to around $10^{19} \mathrm{eV}$ is shown by Iyono in 2011 6. The differential primary energy spectrum has been observed since 2006 [8.

In order to estimate the primary energy of EAS at the ground level, the observation of the core distance and a local particle density are required. It is known that the number of EAS particles and the area arriving them are proportional to the primary energy $E$, on the other hand, the number of EAS events is inversely proportional to the power-law of $E$. Thus typical observation methods of EAS are by using many detectors installed in a large area or sky monitoring detectors such as a fluorescence telescope detector. However, such as a large scale observation increases in cost. Each EAS array in LAAS experiment provides a low cost observation, because compact EAS arrays which basically consist of 8 scintillation counters are used. On the other hand, the observation of a lateral distribution is not enough to estimate the primary energy, because the covered area of our array is about several hundred square meters. In order to solve this problem, we have observed properties of EAS by using Linsley's method 9 . This method is able to be obtained the core distance $r$ by observing EAS arrival time structure. Bezboruah 10 was also reported on the estimation of primary energy spectrum by using the same method. We have adapted the Linsley's method to our compact EAS array observation and have studied the primary energy spectrum since 2006. And in order to improve the determination accuracy of the primary energy, we have installed the apparatus for restricting zenith angle of EAS events in 2010.

In this paper, we report on the relocation and the upgrade of the apparatus.

\section{Apparatus}

The observation site of Okayama University of Science (OUS) is Okayama city, in Japan, and is located at $34^{\circ} 42^{\circ}(\mathrm{N})$ and $133^{\circ} 56^{\circ}(\mathrm{E})$. We have carried out the observation of cosmic rays by using five EAS arrays. Note that we describe the arrays as the OUS1, the OUS2, the OUS3, the OUS4 and the OUS5 in this paper, respectively. Figure 1-(A) shows the campus map of OUS. OUS arrays are typical surface ones set at the ground level, however the arrays are small size. The OUS1 shown in Figure 1-(B), the OUS2 and the OUS3 arrays consist of 8 scintillation counters, and the OUS5 array consists of 5 ones. The OUS4 array is used to obtain zenith angle information of EAS events and is set up scintillation counters as the Figure 2,

\subsection{OUS1 array}

In the OUS1 array, we set 8 scintillation counters on the rooftop in the building of Okayama 

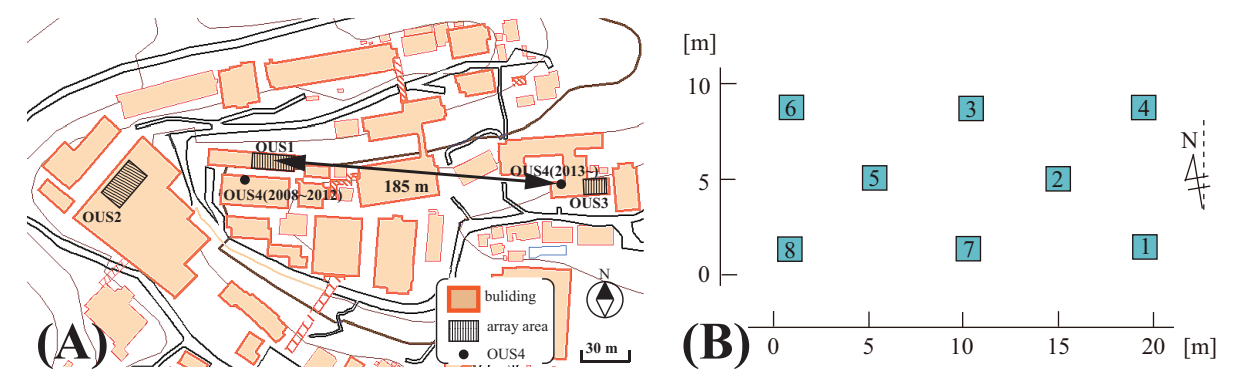

Figure 1: (A) The location of OUS arrays in OUS campus map. The original topographic map is used in Geospatial Information Authority of Japan (GSI) 11, and the campus map is made by modifying the GSI's data. (B) The arrangement of the OUS1. The filled square symbols represent scintillation counters.

University of Science, and show the arrangement in Figure 1-(B). Each scintillation counter equips a Plastic scintillator of which size is $50 \mathrm{~cm} \times 50 \mathrm{~cm} \times 5 \mathrm{~cm}$ and a PMT, and covered a stainless steel container. The covered area of the OUS1 is about $200 \mathrm{~m}^{2}$. The data acquisition system (DAQ) provides a CAMAC TDC, a CAMAC ADC and a shift register. The observation time is obtained by using a GPS module. The time accuracy is $1 \mu \mathrm{s}$ and is maintained by receiving 1 pps GPS signal. The obtained data by the TDC, the ADC and the GPS is stored finally by a Linux PC and data by the shift register is stored by a Windows PC.

An EAS event is triggered by a coincidence module when more than three scintillation counters are hit within $2.5 \mu \mathrm{s}$. In order to measure timing of EAS event signals, a TDC is usually used. However, we need time information of late arriving particles from an air shower front in this work. Since a shift register is able to record a longer time-width in EAS event signals than the TDC, the shift register system used instead of a TDC. The shift register has 16 channels and is able to record timing signal from each scintillation counter. The maximum time-width is $5 \mu$ s and the time resolution is $5 \mathrm{~ns}$ in each channel. The shift register constantly monitors signals from scintillation counters. When the shift register receives an EAS trigger signal from the coincidence module, the shift register records signal data from each scintillation counter within $\pm 2.5 \mu$ s from time of the EAS trigger signal. Data of the shift register is a digital time series data and is not a wave form one.

\subsection{OUS4 array}

We have operated the OUS4 to restrict a large zenith angle EAS event since 2008. By carrying out the synchronized observation between the OUS1 and the OUS4, the restricted data for EAS zenith angle is able to be obtained. The OUS4 had been located at the distance of about $10 \mathrm{~m}$ from the OUS1 in the observation term between 2008 and 2012. We moved the OUS4 to new site that was located at the distance of about $180 \mathrm{~m}$ from the OUS1 in 2013, and after a pilot study and maintenance, we have been making improvement to the OUS4 since 2016. In this paper, the OUS4 from 2008 to 2012 is described as the OUS4-ver.1 and new OUS4 is described as the OUS4-ver.2, respectively. And the synchronized observation between the OUS1 and the OUS4-ver.(1,2) is also described as the OUS1+4-ver.1 and the OUS1+4-ver.2, respectively.

The schematic drawing of the OUS4-ver.2 is shown in Figure 2, The OUS4-ver.2 equips one 
scintillation counter for EAS event trigger on the top and bottom, respectively. At the side panels, 4 anti-counters are installed in the vertical direction of the trigger counters. Each counter is the same as that of the OUS1, and its size is $50 \mathrm{~cm} \times 50 \mathrm{~cm} \times 5 \mathrm{~cm}$. DAQ and the GPS are also the same as the OUS1. The synchronized observation is carried out by using the GPS time.

In the modification from the OUS4-ver.1 to the OUS4-ver.2, the area of the trigger counters increases to $0.25 \mathrm{~m}^{2}$ from $0.2 \mathrm{~m}^{2}$, and the distance between the trigger counters decreases to $1150 \mathrm{~mm}$ from $1350 \mathrm{~mm}$. Thus the restriction zenith angle $\theta$ for the EAS event is modified to $\theta=30^{\circ}$ from $\theta=26^{\circ}$.

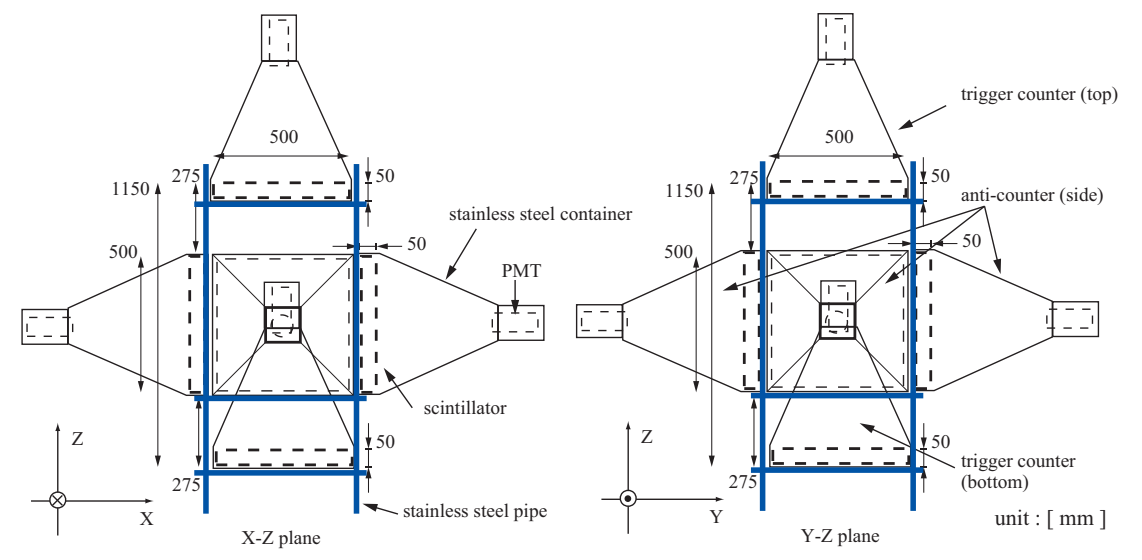

Figure 2: The schematic drawing of the OUS4-ver.2.

\section{Simulation result}

In the estimation for the EAS core distance $r$, we have applied the Linsley's method to our observation. Additionally, we have carried out the simulation for detectors and making database of the lateral distribution, and have combined the observed data and simulated results. The EAS simulation program Aires 12] is used. QGSJET II-3 [13 and Hillas Splitting model 14 are assumed to be for hadronic interaction models. Protons and Irons are assumed to be for primary particles, respectively. The primary energy $E_{0}$ are assumed to be in its energy range of $10^{15} \mathrm{eV}$ to $10^{20} \mathrm{eV}$. The detector simulation and making the database of the lateral distribution are described in detail in the reference [8, 15]

\subsection{Core distance}

The dependence of the core distance $r$ to the dispersion $\sigma_{\mathrm{t}}$ of the arrival time distribution of each EAS's particles has been shown experimentally by Linsley. Linsley described the average dispersion $\left\langle\sigma_{\mathrm{t}}\right\rangle$ of observed events as following

$$
\left\langle\sigma_{t}\right\rangle=\sigma_{t 0}\left(1+\frac{r}{r_{t}}\right)^{b}
$$

where $\sigma_{t 0}=1.6 \mathrm{~ns}, r_{t}=30 \mathrm{~m}, b=(2.08 \pm 0.08)-(0.4 \pm 0.06) \sec \theta+(0 \pm 0.06) \log \left(E / 10^{17} \mathrm{eV}\right)$, $\theta$ and $E$ are the zenith angle and the primary cosmic ray energy, respectively. Since we are not 
able to detect the zenith angle of each EAS event, we use $b$ averaged by the expected zenith angle distribution in this observation, and ignore the term of the primary energy. The relation of $r$ and $\sigma_{\mathrm{t}}$ is plotted in Figure 3 .

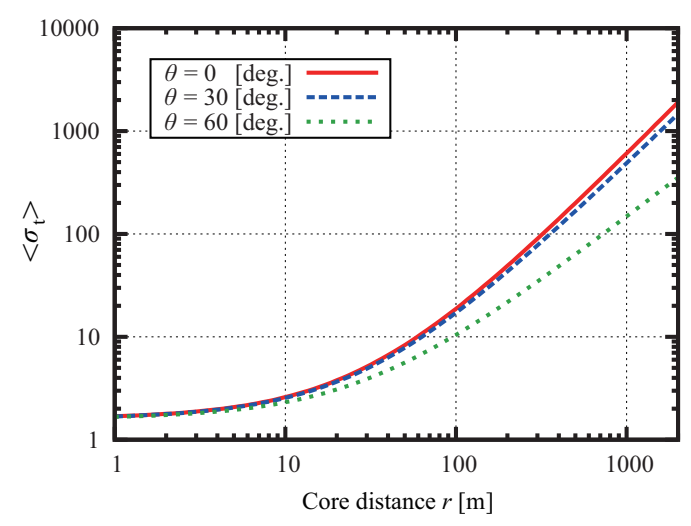

Figure 3: The relation between $r$ and $\sigma_{\mathrm{t}}$ in $\theta=0^{\circ}, 30^{\circ}, 60^{\circ}$.

The arrival time distribution of EAS particles is assumes to be the gamma distribution, and the median value $t_{\mathrm{m}}$ of the distribution is applied to the best estimator of the dispersion. The discussion of the estimator is described in Okita 7. Thus, $\sigma_{t}$ is shown as

$$
\sigma_{\mathrm{t}}=\frac{\sqrt{2}}{1.67} t_{\mathrm{m}}
$$

By using equation 3.1 and equation 3.2, we are able to calculate $r$ as

$$
r=30\left(\left(1.35 t_{\mathrm{m}}\right)^{\frac{1}{1.65}}\right)
$$

In the core distance $r$, we focus events of $r>100 \mathrm{~m}$ and carry out data analysis, because the relative error of $r$ in $r<100 \mathrm{~m}$ is greater than that of $r$ in $r>100 \mathrm{~m}$.

\subsection{Acceptance}

Figure 4 shows the comparison of the OUS1 with the OUS1+4-ver.2 in the acceptance obtained by the simulation. The acceptance of the OUS1+4 is about $1 / 100$ times that of the OUS 1 at the input primary energy $E_{0}=10^{16} \mathrm{eV}$, and is about $1 / 30$ times that of the OUS1 at $E_{0}=10^{19} \mathrm{eV}$.

Figure 5 shows comparisons of the OUS1+4-ver. 1 with the OUS1+4-ver.2 in the acceptance in proton and iron primary nuclei, respectively. The primary energy range above $10^{17} \mathrm{eV}$ in the proton primary, the acceptance of the OUS1+4-ver. 2 is about $20 \%$ greater than that of OUS1+4-ver.1, and above $10^{19} \mathrm{eV}$, that of the OUS1+4-ver.2 is about $30 \%$ greater than that of OUS1+4-ver.1. The acceptance deceases with an increase of the distance between the OUS1 and the OUS4 due to the coincidence trigger probability, however, the contribution of the distance is small, because the EAS event criterion for the core distance $r$ is originally $r>100 \mathrm{~m}$. In the iron primary, a similar trend is shown. 


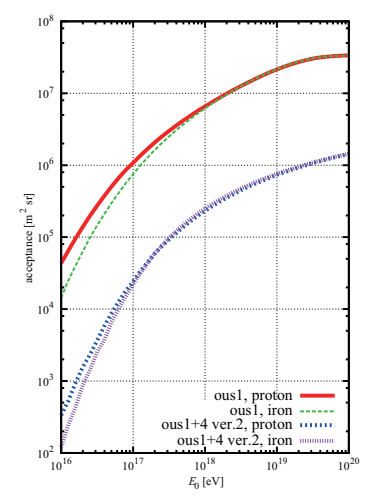

Figure 4: The comparison of the acceptance of the OUS1 with OUS1+4-ver.2.
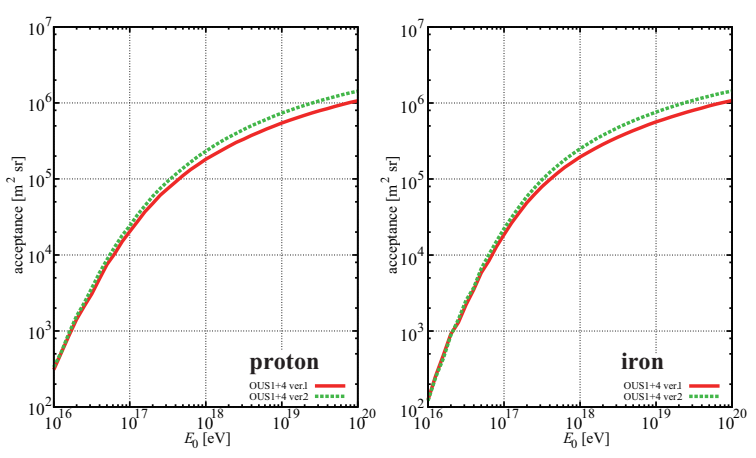

Figure 5: Comparisons of the acceptance of the OUS1+4-ver.1 with OUS1+4-ver.2 in primary protons and irons, respectively.

\subsection{Primary energy determination}

The simulated primary energy distribution obtained by each array observation system, when the input primary energy is $E_{0}=10^{17} \mathrm{eV}$, is shown in Figure 6 By restricting the EAS zenith angle, the FWHM of obtained primary energy distribution in the OUS1+4 is smaller than that of the OUS1. The procedure method is described in Reference [8].

Figure 7 is shown the comparison of the OUS1+4-ver.1 with the OUS1+4-ver.2. $E_{1}$ and $E_{\mathrm{h}}$ indicate both ends of the FWHM of the simulated energy distribution, respectively. Each FWHM value of the OUS1+4-ver.1 and the OUS1+4-ver.2 is $10^{16.4} \mathrm{eV}-10^{17.3} \mathrm{eV}$ and $10^{16.3} \mathrm{eV}-$ $10^{17.3} \mathrm{eV}$,respectively, in the primary proton at $E_{0}=10^{17} \mathrm{eV}$. Since the restriction of the zenith angle $\theta$ in the OUS1+4-ver.2 is $\theta=30^{\circ}$, the FWHM of the OUS1+4-ver.2 become slightly greater than that of the OUS1+4-ver.1 in the low energy region.

The FWHM values of the simulated energy distribution are plotted as the systematic error in the determination of the primary energy in Figure 8 and Figure 9 , respectively.

\section{Conclusion}

The primary energy spectrum has been studied by using compact EAS arrays and the Linsley's 

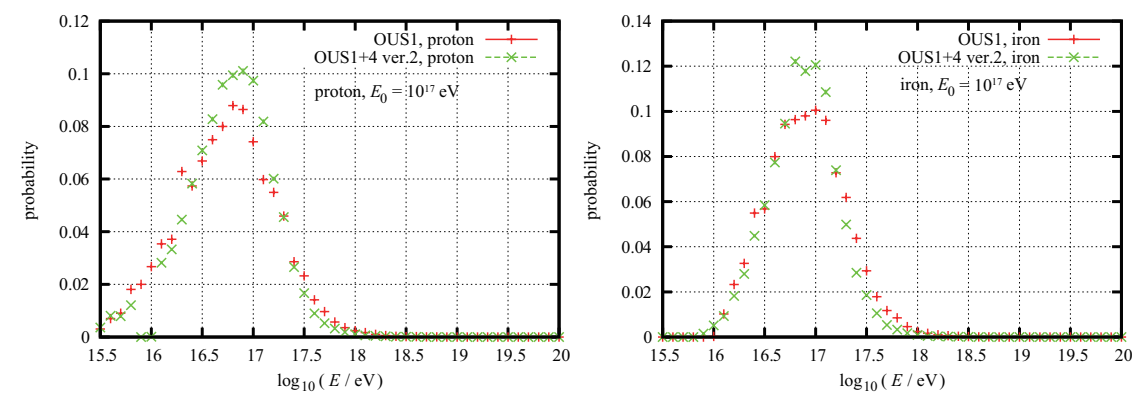

Figure 6: Comparisons of the simulated energy distribution of the OUS1 with the OUS1+4-ver.2 in primary protons and irons, respectively.
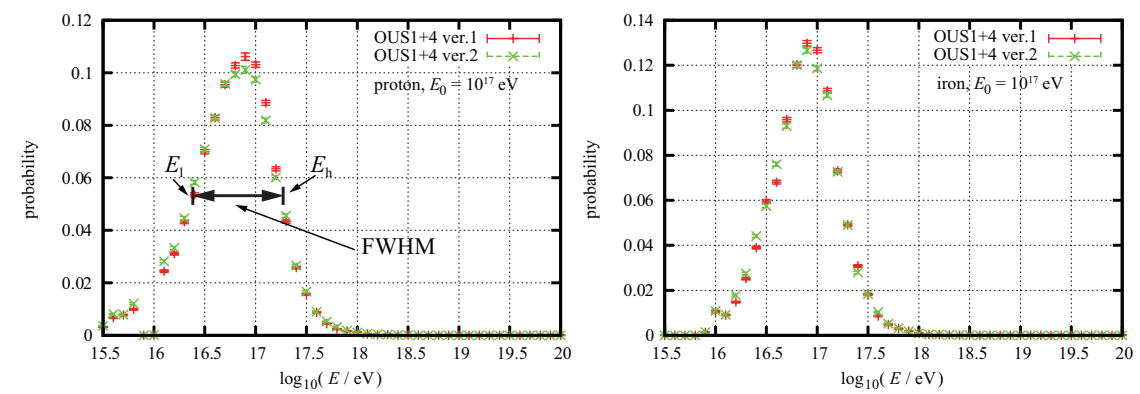

Figure 7: Comparisons of the simulated energy distribution of the OUS1+4-ver.1 with the OUS1+4-ver.2 in primary protons and irons, respectively.
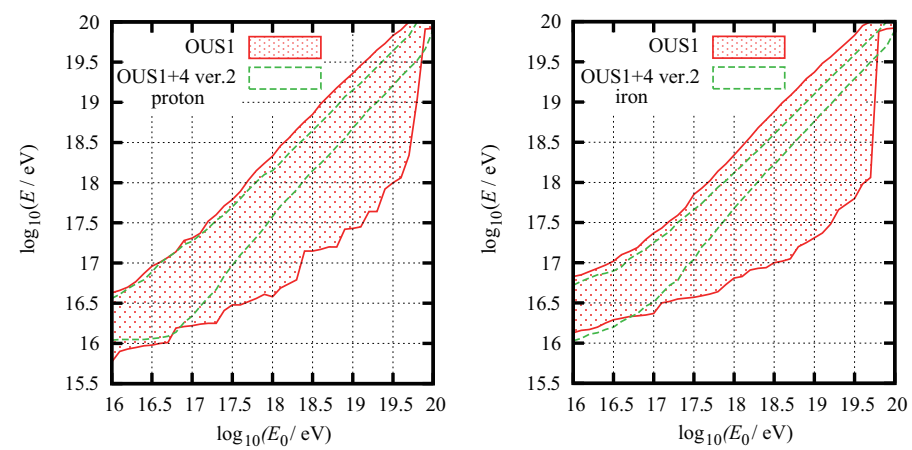

Figure 8: In the the systematic error of the determination of $E$, comparisons of that of the OUS1 with that of the OUS1+4-ver.2 in primary protons and irons, respectively.

method in LAAS experiments since 2006. The simulation for the relocation and the upgrade of the OUS4 array is performed, and the improvement of the acceptance of the OUS1+4-ver.2 is shown. The difference of the systematic error in the primary energy determination is small in the comparison of the OUS1+4-ver.1 with the OUS1+4-ver.2. We will carry out continuous observation with the OUS4 and obtain more statistics of EAS data. 

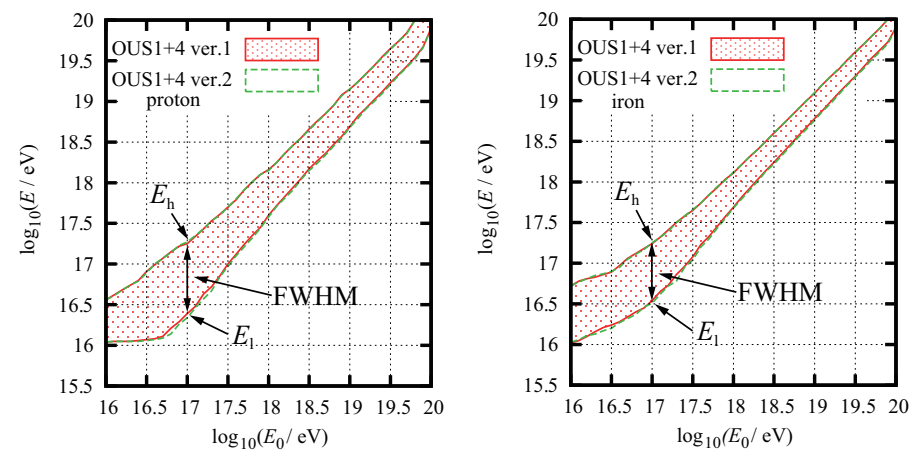

Figure 9: In the the systematic error of the determination of $E$, comparisons of that of the OUS1+4-ver.1 with that of the OUS1+4-ver.2 in primary protons and irons, respectively.

\section{References}

[1] T. Wada et al., Observation of time correlation, Nucl. Phys. B (Proc. Suppl.), 1999, 75(issue 1-2) 330-332

[2] N. Ochi et al., Search for large-scale coincidences, J. Phys. G: Nucl. Part. Phys., 2003, 29 1169-1180

[3] N.M. Gerasimova and G.T. Zatsepin, Disintegration of Cosmic-Ray Nuclei , JETF, 1960, 11, 4, 899

[4] A. Iyono et al., Observation of Cosmic Ray Anisotropy, in proceedings of 30th ICRC Merida, 2007, 114

[5] S. Ohara et al., The Anisotropy of Cosmic Ray, in proceedings of 32nd ICRC Beijing, 2011, 406

[6] A. Iyono et al., Cosmic ray composition studies, Astrophysics and Space Sciences Transactions, 2011, $7327-333$

[7] M. Okita et al., Primary energy spectrum of, Nucl. Phys. B (Proc. Suppl.), 2008, 175-176, 322-325

[8] H. Matsumoto et. al., Simulation studies and implementation, Nucl. Instr. \& Meth. A, 2010, 614 $475-482$

[9] J. Linsley, Thickness of the particle, J. Phys. G: Nucl. Phys., 198612 51-57

[10] Bezboruah et al., A non conventional method, Astropart. Phys., 1996 11, 395

[11] Geospatial Information Authority of Japan, www.gsi.go.jp

[12] S. J. Sciutto, AIRES, 1999, [astro-ph/9911331]

[13] S. Ostapchenko, Nonlinear screening effects, Phys. Rev. D, 2006, 74014026

[14] A. M. Hillas, Shower simulation, Nucl. Phys. B (Proc. Suppl.), 1997, 52(issue 3) 29-42

[15] H. Matsumoto et. al., The primary energy spectrum, in proceedings of 34th ICRC Hague, 2015 